\title{
RECRIA DE RÃ-TOURO (RANA CATESBEIANA) EM TANQUES- REDE ALOJADOS EM VIVEIROS DE TILÁPIA
}

\author{
RAISING BULLFROGS (RANA CATESBEIANA) IN CAGES PLACED IN TILAPIA PONDS \\ Sousa, R.M.R. ${ }^{1 *}$, Agostinho, C.A. ${ }^{1 \mathrm{~A}}$, Oliveira, F.A. ${ }^{1 \mathrm{~B}}$, Argentim, D. ${ }^{1 \mathrm{C}}$, Oliveira, L.C. ${ }^{1 \mathrm{D}}$, \\ Wechsler, F.S. ${ }^{1 E}$ e Agostinho, S.M.M. ${ }^{1 F}$
}

${ }^{1}$ Departamento de Produção Animal. Universidade Estadual Paulista. CP 560-Fazenda Lageado/FMVZUNESP. Campus de Botucatu. CEP 18618-000. Botucatu-SP. Brasil. 1*romorgado@fmvz.unesp.br; Aagostinho@fca.unesp.br; Bfao3@hotmail.com; Cfelis_bino@hotmail.com; Dcaetanopesca@hotmail.com; Ewechsler@fca.unesp.br; Fagostinho@fca.unesp.br

\section{PalaVRas CHAVE ADICIONAIS}

Ranicultura.

\section{RESUMO}

No Brasil, os sistemas de criação de rãs (confinamento, anfigranja, inundado) utilizam um volume de água insuficiente para a manutenção da estabilidade térmica, e a baixa renovação prejudica a qualidade da água. Neste trabalho foi proposto a criação de rãs em tanques-rede distribuídos em viveiros com grande estabilidade térmica. $O$ experimento consistiu em avaliar o desempenho produtivo de rãs-touro alojadas em tanques-rede de $1 \mathrm{~m}^{3}$, nas densidades de: 28,56 e 84 animais por tanque-rede, alimentadas com ração fornecida por dispensador automático. Nos viveiros de $72 \mathrm{~m}^{2}$ utilizaram-se duas densidades de tilápias (200 e 300 peixes por viveiro). O período experimental foi de 105 dias para as tilápias (maio a agosto) e de 60 dias para as rãs (julho e agosto). Não foram observadas diferenças significativas no desempenho das rãs para as diferentes densidades. A utilização de 200 tilápias por viveiro melhorou o ganho de peso dos peixes e a concentração de oxigênio dissolvido da água, comparado com a densidade de 300 peixes por viveiro. Este novo sistema de criação proporcionou um ambiente adequado para as rãs com ganho de peso e ausência de mortalidade durante o inverno.

\section{SUMMARY}

Brazilian systems for raising bullfrogs (concrete enclosure, concrete enclosure with feed dispenser and shelter, flooded enclosure) do not use enough water to keep temperature stable, and

Recibido: 6-12-07. Aceptado: 1-4-08.

\section{AdDitionAl KEYWORDS}

Frog raising.

the low rate of water renewal hinders water quality. In this article we propose raising frogs in cages placed in fish ponds having high thermal stability. The experiment consisted in evaluating the productive performance of bullfrogs housed in $1 \mathrm{~m}^{3}$ cages at the densities of 28,56 or 84 animals/ cage, fed a ration delivered by an automatic dispenser. Each pond was $72 \mathrm{~m}^{2}$ large. Two fish densities were used (200 or 300 tilapias/pond). The experimental period lasted 105 days for tilapias (May-August) and 60 days for frogs (July-August). No significant differences among densities were observed for frog performance. Using 200 tilapias/ pond improved fish weight gain and dissolved oxygen concentration, as compared to 300 tilapias/ pond. This new rearing system provided an adequate environment for frogs, as there was weight gain and zero mortality during winter.

\section{INTRODUÇÃO}

A rã-touro, originária da América do Norte, foi importada e introduzida nos ranários comerciais na década de 30 (Ranário Aurora, 1938) e adaptou-se ao clima brasileiro, apresentando rusticidade e alcançando a maturidade sexual em menos de um ano. Assim, iniciou-se o processo do desenvolvimento da tecnologia de criação de rãs que, atualmente ainda demonstra estar em fase de consolidação tecnológica 
(Lima et al., 2003), tornando fundamentais as pesquisas envolvam manejo de criação. A produção de rãs no Brasil é de aproximadamente 300 toneladas por ano e os ranicultores utilizam pelo menos um dos três sistemas de criação (Confinamento, Anfigranja e Inundado) descritos por Lima et al., 1999.

Nos três sistemas o volume de água utilizado em cada baia de recria é pequeno, o que pode levar à grandes oscilações térmicas do dia para a noite (Hollman, 1998). Além disso, o pequeno volume de água associado à alta densidade de animais facilita a proliferação de bactérias patogênicas devido à grande quantidade de fezes e restos de ração. Segundo Casali et al. (2005) o acúmulo de ração na água e as maiores quantidades de fezes em altas densidades causam baixo desempenho e pioram as condições higiênicas da baia, recomendando novas pesquisas com manejo de troca de água e métodos para reduzir o desperdício de ração e manter a qualidade do ambiente.

Na aqüicultura mundial o consórcio e o policultivo visam a eficiente ocupação do espaço físico e dos diferentes nichos alimentares dos viveiros, proporcionando aumento da produtividade e rentabilidade dos empreendimentos aqüícolas, com um pequeno gasto adicional e com ganhos ambientais. Um exemplo é a criação de camarões com tilápias. Devido ao hábito alimentar das tilápias e ao possível controle da mancha branca, aliado a melhoria na qualidade da água e do solo, esta modalidade de cultivo desempenha papel positivo e benéfico para o aumento da sobrevivência dos camarões nos viveiros (Mello e Farias, 2007). Além do consórcio com camarões, a tilápia, por ser onívora e não predar anfíbios, poderá ter êxito na criação conjunta com rãs, se alimentando das sobras de ração.

Neste sentido, o presente trabalho teve como objetivo avaliar o desempenho produtivo de rãs-touro em tanques-rede alojados em viveiros, povoados com tilápias.

\section{MATERIAL E MÉTODOS}

O experimento foi conduzido em seis viveiros medindo $72 \mathrm{~m}^{2}$ e em cada viveiro foram colocados três tanques-rede (figura 1) com diferentes densidades de rãs: 28, 56 e 84 animais. A densidade máxima dentro de cada tanque-rede foi baseada na densidade utilizada nos ranários comerciais onde, dependendo do sistema de criação, pode variar de 50 a 100 animais. Os tanques-rede (figura 1) com 1,0 metro de largura, 1,0 metro de comprimento e 1,2 metro de altura foram confeccionados com malhas de tela metálica 5/8" (16 mm), recoberta com PVC. O tamanho da malha de $16 \mathrm{~mm}$ foi suficiente para evitar fuga de imagos com pesos superiores a $25 \mathrm{~g}$. A tampa com estrutura metálica e recoberta com tela, desliza sobre um trilho, permitindo a abertura gradativa que evita a fuga dos animais durante o manejo. Uma moldura confeccionada com cano de PVC de $1 / 2$ ", recoberta com tela metálica, foi fixada nas laterais do tanque-rede de modo a formar uma plataforma telada sobre a qual era possível ajustar uma lamina de água de 2 a $5 \mathrm{~cm}$ de acordo com o tamanho da rã. Um abrigo com $3 \mathrm{~cm}$ de altura e $30 \mathrm{~cm}$ de largura foi colocado em uma das extremidades do tanque rede.

O arraçoamento diário foi realizado baseando-se no peso vivo das rãs de acordo com a tabela proposta por Lima et al. (2003). $\mathrm{O}$ alimento foi oferecido de hora em hora utilizando um dispensador automático de ração que consiste em um reservatório cônico contendo um mecanismo eletromecânico que libera ração em intervalos pré-estabelecidos por temporizadores. Confeccionado com material impermeável (fibra de vidro) o reservatório possui uma saída em forma de funil. O movimento de rotação para a liberação dos peletes é promovido por um motor com caixa de redução da rotação. O alimento é despejado no interior de cada tanque rede. A quantidade oferecida foi ajustada quinzenalmente com base no peso vivo dos animais. A ração

Archivos de zootecnia vol. 59, núm. 225, p. 32. 
A) Corte longitudinal do viveiro

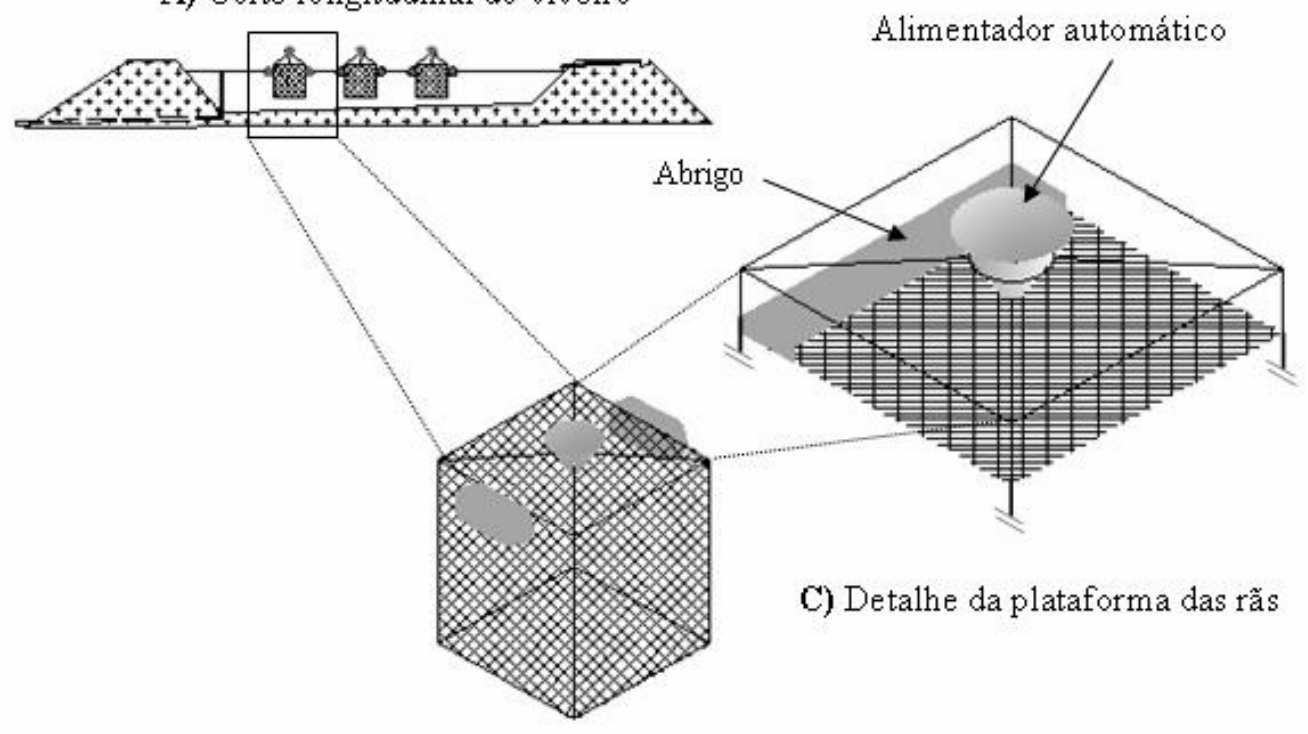

B) Vista geral do tanque-rede

Figura 1. Disposição dos tanques-rede no viveiro e detalhe do tanque-rede com a plataforma para as rãs. (Arrangement of the cages in the ponds, with a detailed view of a cage with its platform for frogs).

(peletes de 5,0 a 8,0 mm) era constituída, segundo o fabricante, de $40 \%$ de $\mathrm{PB}, 13 \%$ de Umid, $4 \%$ de EE, $6 \%$ de MF, $12 \%$ de MM, $2,5 \%$ de Ca e $0,8 \%$ de $P$.

Devido à desuniformidade dos lotes provenientes de baias semelhantes as do sistema anfigranja, as rãs foram distribuídos em blocos casualizados em duas faixas de peso: bloco $1=77,0 \mathrm{~g} \pm 4,0 \mathrm{~g}$ e bloco $2=140,0$ $\mathrm{g} \pm 26,0 \mathrm{~g}$. Foram utilizados juvenis de tilápia do Nilo (Oreochromis niloticus) com peso médio de 32,0 $\mathrm{g} \pm 2,7 \mathrm{~g}$ distribuídos nos seis viveiros, sendo três com 300 tilápias (D300) e três com 200 tilápias (D200). As tilápias permaneceram no experimento por um período de 105 dias (maio a agosto). Nos meses de maio e junho, período em que ainda não havia rãs nos tanques-rede, as tilápias foram alimentadas de hora em hora por meio dos dispensadores automáticos com a mesma ração do experimento, na porcen- tagem de 3\% do peso vivo. Nos meses de julho e agosto (60 dias) as tilápias consumiram somente as sobras provenientes dos tanques-rede com rãs.

As temperaturas máxima e mínima, pH e OD da água foram registradas diariamente. Para a determinação da amônia dissolvida, a água de cada viveiro foi coletada ao final do período experimental com uma garrafa de 0,5là 30,0 cm de profundidade e mantida em freezer a $-20^{\circ} \mathrm{C}$ até o momento da filtração. O volume filtrado foi destinado para a análise por espectrofotometria, no Laboratório de Limnologia do Instituto de Biociências da Unesp de Botucatu. Especialmente neste experimento não foi necessário realizar manejos de aumento de renovação de água, ou de diminuição da oferta de alimento, em função de eutrofização do meio.

O delineamento experimental foi realizado em blocos casualizados com parcelas 
subdivididas. O peso final e o ganho de peso diário dos animais variaram em função das densidades de rãs-touro (3 tratamentos em 2 blocos), das densidades de tilápias (2 parcelas) e das interações entre densidade de rãs e tilápias e foram submetidos à análise de variância, seguida do teste de Tukey, adotando-se o nível de 5\% para avaliar a significância.

\section{RESULTADOSEDISCUSSÃO}

Nos sistemas de criação, utilizados atualmente, verificam-se altas taxas de mortalidade em locais que ocorrem invernos rigorosos, e o crescimento dos animais é interrompido. Apesar das baixas temperaturas observadas nos meses frios durante este experimento $\left(18,1^{\circ} \mathrm{C}\right.$ média geral) não ocorreu mortalidade e houve crescimento das rãs e das tilápias em todos os tratamentos (tabela I). Este sistema pode ser também uma alternativa, sem perdas, para rãs que atrasem o tempo de engorda e necessitem atravessar
Tabela I. Crescimento de rãs-touro criadas em tanques-rede alojadas em viveiros com tilápias durante o inverno (PI= peso inicial; $P F=$ peso final; $G P D=$ ganho de peso diário). (Growth rate of frogs housed in cages placed in tilapia ponds during winter $(\mathrm{PI}=$ initial weight; $\mathrm{PF}=$ final weight; GPD= daily weight gain)).

\begin{tabular}{lccc}
\hline Densidade & PI (g) & PF (g) & GPD (g) \\
\hline 200 tilápias & $32,4 \pm 2,5$ & $232,6 \pm 57,9^{A}$ & $1,91 \pm{ }^{\mathrm{A}}$ \\
300 tilápias & $32,7 \pm 2,9$ & $160,0 \pm 50,8^{\mathrm{B}}$ & $1,22 \pm{ }^{\mathrm{B}}$ \\
28 rãs & $105,4 \pm 34,1$ & $139,2 \pm 54,4^{*}$ & $0,56 \pm$ * $^{*}$ \\
56 rãs & $105,8 \pm 35,9$ & $130,4 \pm 53,2^{*}$ & $0,40 \pm$ * $^{*}$ \\
84 rãs & $104,1 \pm 33,8$ & $129,3 \pm 42,6^{*}$ & $0,42 \pm{ }^{*}$ \\
\hline
\end{tabular}

Médias seguidas de letras diferentes, na coluna, diferem entre si pelo teste de Tukey a $5 \%$ de probabilidade. (*) Não significativo.

o inverno. Entretanto, os ganhos médios de peso das rãs nas diferentes densidades não apresentaram diferenças ( $p>0,05)$, pois nesta época do ano o metabolismo dos animais pecilotérmicos é baixo, conseqüentemente

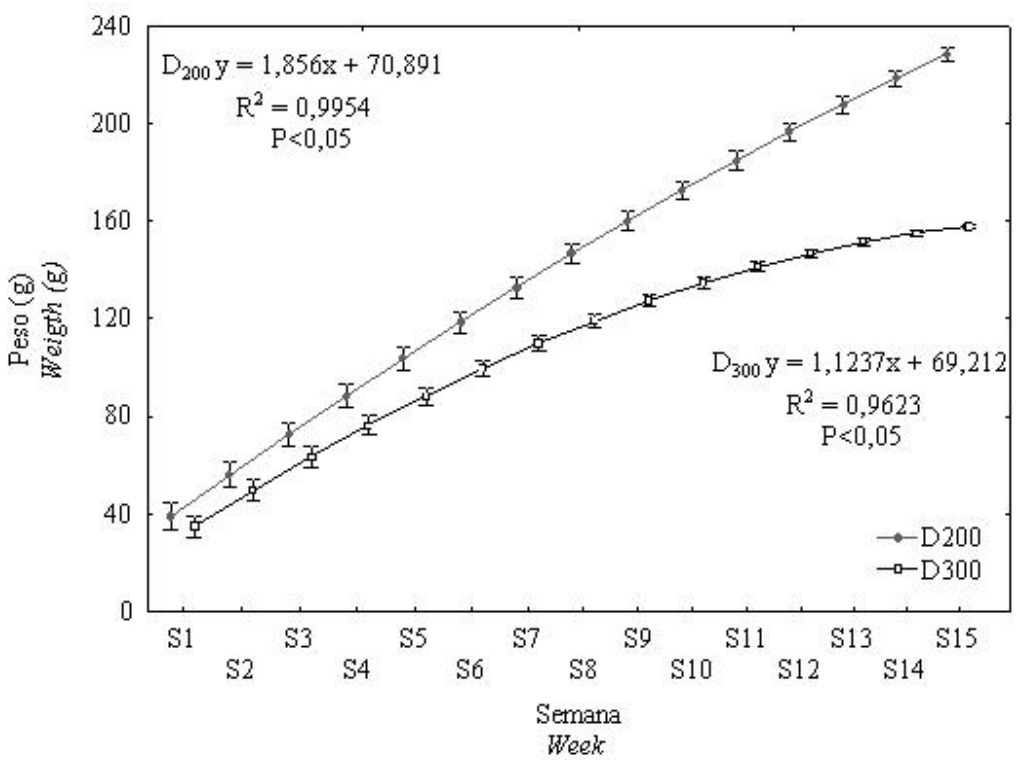

Figura 2. Curva de crescimento das tilápias no período experimental. (Growth curve of frogs during the experimental period).

Archivos de zootecnia vol. 59, núm. 225, p. 34. 
não houve competição por alimento e por espaço, discordando do desempenho de rãs criadas no sistema anfigranja observado por Casali et al.(2005). Provavelmente, em função do retardo no crescimento dos animais, o período experimental utilizado tenha sido curto demais para que as diferenças entre tratamentos fossem observadas. Sugere-se que densidades mais altas possam ser testadas e utilizadas no sistema de tanques-rede, aproveitando melhor a área de cada unidade de produção em experimentos de longa duração.

Entre os parâmetros abióticos da água, pode-se destacar a temperatura como principal fator a influenciar os processos metabólicos dos animais pecilotérmicos que, tem sua digestão afetada pela temperatura ambiente em pelo menos cinco processos: consumo de alimento, nível de secreção de sucos digestórios, atividade enzimática, motilidade do trato gastrintestinal e taxa de absorção intestinal (Baldisserotto, 2002). Segundo Mello (2001) as rãs não ganham peso quando a temperatura ambiente é menor do que $18^{\circ} \mathrm{C}$.

Na figura 2, observa-se que o ganho de peso das tilápias do tratamento com maior densidade foi inferior ao das tilápias com menor densidade $(\mathrm{p}<0,05)$, corroborando o desempenho de tilápias observado por Vieira et al. (2000) e Kubitza (2000) e discordando dos valores observados por Carneiro et al. (1999). O experimento foi conduzido nos meses de inverno (maio, junho, julho e agosto) e, a partir do início do mês de agosto a diferença no ganho médio de peso das tilápias entre os tratamentos foi mais evidente. Tendo o tratamento de menor densidade um melhor desempenho. Isto provavelmente deve ter ocorrido, pois no mês de agosto a temperatura média foi maior, melhorando o consumo. Entretanto, para as duas densidades de tilápias a sobra de alimento foi a mesma, este fator passou a ser limitante no ganho de peso das tilápias em maior densidade.

Para a avaliação da qualidade da água

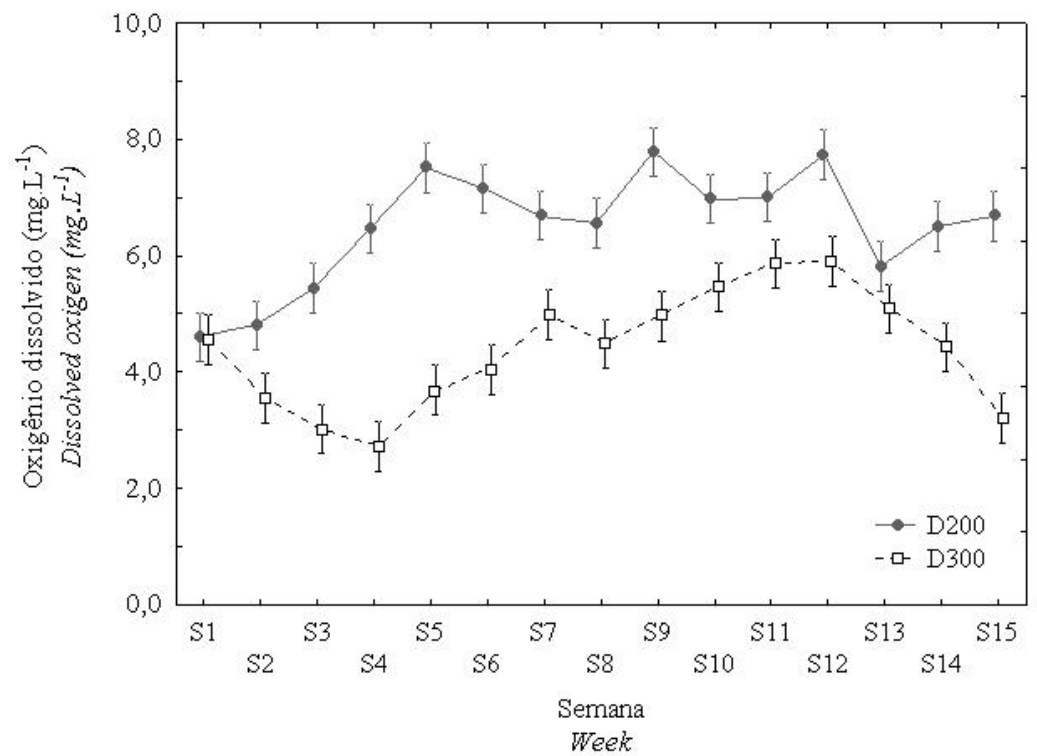

Figura 3. Valores médios de oxigênio dissolvido durante o período experimental. (Average values of water dissolved oxygen during the experimental period). 
observaram-se as alterações da quantidade de OD e de $\mathrm{pH}$ no decorrer do experimento. A disponibilidade de OD na água no período experimental apresentou diferenças significativas com interação entre o tratamento e período (semana) $(\mathrm{p}<0,05 ; \mathrm{F}=13,44)$, delineando uma relação de aumento da biomassa e redução do OD, sendo que o tratamento D300 respondeu por uma maior inferência na redução do OD na água, apresentando alta redução no mês de agosto (S13, S14 e S15) (figura 3). Ressalta-se que níveis de oxigênio dissolvido na água abaixo de 5 $\mathrm{mg} / \mathrm{l}$ podem prejudicar o desempenho produtivo de tilápias do Nilo (Kubitza, 2000).

Os viveiros de 300 tilápias mesmo contendo uma quantidade de peixes 50\% superior apresentaram ao final do experimento uma biomassa média total de 48,0 kg e os viveiros de 200 tilápias 46,6 kg. Esta diferença é pequena, mas o peso individual dos peixes do tratamento D300 foi inferior ao dos peixes do tratamento D200 (figura 3), acarretando um maior consumo de OD no tratamento D300. Segundo Arana (1997) peixes menores (da mesma espécie) apresentam maior consumo de OD do que peixes maiores. Para os valores de $\mathrm{pH}$ não foram observadas diferenças entre os tratamentos, entretanto diferindo no tempo, igualmente para as duas densidades de tilápias $(p<0,05 ; F=6,0)$, tendendo à alcalinidade do início para o fim do experimento $(6,9-7,1)$.

No período experimental a variação da temperatura da água superficial foi grande e praticamente a mesma para os tratamentos, diferindo do início para o final do experimento ( $<<0,05 ; \mathrm{F}=23,22)$, onde os menores valores foram registrados nas primeiras semanas de julho (S7; S8) (figura 4) diferindo do mês de agosto (S13; S14; S15) que apresentou as maiores médias, o que contribuiu para o melhor desempenho neste período. Ressalta-se que temperaturas baixas reduzem o metabolismo dos animais pecilotérmicos, resultando em menor consumo de alimento, menor ganho de peso, baixando a resposta imunológica, podendo acarretar mortalidade (Boujard e Leatherland, 1992; Meer et al., 1997; Toguyeni et al., 1997; Urbinati e Carneiro, 2004).

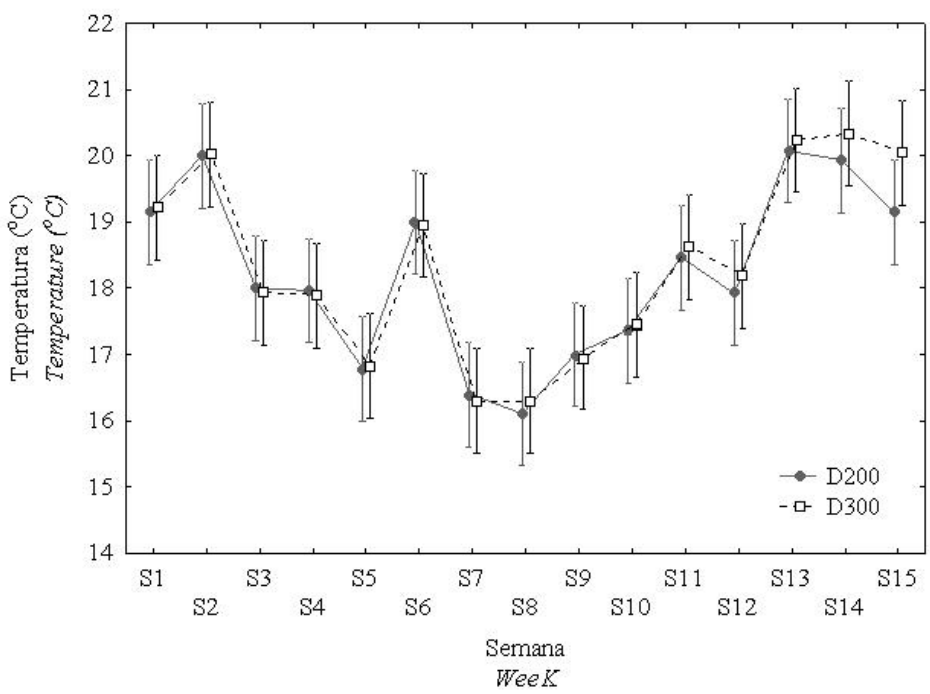

Figura 4. Valores médios de temperatura da água durante o período experimental. (Average values of water temperature during the experimental period).

Archivos de zootecnia vol. 59, núm. 225, p. 36. 


\section{RECRIA DE RÃ-TOURO EM TANQUES-REDE ALOJADOS EM VIVEIROS DE TILÁPIA}

A concentração média final de amônia tanto dos viveiros com maior densidade quanto nos viveiros com menor densidade foi elevada, 0,68 e $0,62 \mathrm{mg}$ de $\mathrm{NH}_{3} / \mathrm{l}$, respectivamente. Essa concentração elevada nos viveiros pode ser devido à alta taxa de arraçoamento diário, seguido de um menor consumo devido às baixas temperaturas e da baixa renovação de água dos viveiros (5\% de renovação diária). Porém, Kubitza (2000) não observou efeito adverso em tilápia híbrida após exposição por 96 horas a uma concentração de amônia de $0,84 \mathrm{mg} / \mathrm{l}$.

Ressalta-se que o volume de água para as rãs foi superior ao encontrado nos outros sistemas de cultivo o que proporciona melhor qualidade e estabilidade da água utilizada no cultivo. Outro fator que influência a eficiência dos sistemas de criação de rãs é a oferta de alimento. No sistema inundado, boa parte da ração oferecida afunda antes que as rãs possam consumi-la; no sistema de confinamento e no sistema anfigranja a ração é fornecida uma vez ao dia, perdendo qualidade com o tempo. A utilização de tanques-rede com dispensadores automáticos

\section{BIBLIOGRAFIA}

Arana, L.V. 1997. Princípios químicos de qualidade da água em aqüicultura. $1^{\mathrm{a}}$ ed. Florianópolis. Santa Catarina. 165 pp.

Baldisserotto, B. 2002. Fisiologia de peixes aplicada à piscicultura. Ed. UFSM. Santa Maria. 212 pp.

Boujard, T. and Leatherland, J.F. 1992. Demandfeeding behavior and diel pattern of feeding activity in Oncorhynchus mykiss held under different photoperiod regimes. J. Fish Biol., 40, 535-544.

Carneiro, P.C.F., Cyrino, J.E.P. e Castagnolli, N. 1999. Produção da tilápia vermelha da Flórida em tanques-rede. Sci. Agric., 56: 3.

Casali, A.P., Moura, O.M., Mendes, R.R.B. e Campos, V.M. 2005. Efeito da densidade de estocagem no desempenho de rã-touro (Rana catesbeiana) em recria. Rev. Bras. Zootecn., 34: 1828-1834.

Hollman, A. 1998. Perfil térmico de instalações do de ração, na criação de rãs, poderá proporcionar vantagens significativas no desempenho produtivo dos animais, contribuindo para a sustentabilidade da atividade.

\section{CONCLUSÕES}

Os resultados obtidos neste experimento nos permitem concluir que a utilização de tanques-rede proporciona um ambiente adequado à recria de rãs, com ausência de mortalidade e ganho de peso durante o inverno, tornando a utilização deste sistema uma alternativa em ranários que possuam viveiros. Além disso, pode fornecer uma receita adicional com as rãs para piscicultores que utilizam viveiros de criação de tilápias. Entretanto, ainda serão necessárias pesquisas relacionadas às demais fases de crescimento das rãs, freqüência alimentar e a densidade de ambas as espécies durante outras estações do ano.

\section{AGRADECIMENTOS}

Os autores agradecem à FAPESP pelo aporte financeiro.

sistema anfigranja com diferentes alternativas construtiva para a criação da rã-touro (Rana catesbeiana). Dissertação (Mestrado em Engenharia Agrícola). Universidade Federal de Viçosa. 94 pp.

Kubitza, F. 2000. Tilápia, Tecnologia e planejamento na produção comercial. Editora. Acqua Supre Com. Suprim. Aqüicultura Ltda. Jundiaí, SP. 289 pp.

Lima, S.L., Cruz, T.A. e Moura, O.M. 1999. Ranicultura: Análise da cadeia produtiva. UFV. Editora Folha de Viçosa. Viçosa, MG. 172 pp.

Lima, S.L., Casali, A.P. e Agostinho, C.A. 2003. Desempenho zootécnico e percentual de consumo de alimento de rã-touro (Rana catesbeiana) na fase de recria (pós-metamorfose) do sistema anfigranja. Rev. Bras. Zootecn., 32: 505-511.

Meer, M.B.V., Herwaarden, H. and Verdegem, M.C.J. 1997. Effect of number of meals and

Archivos de zootecnia vol. 59, núm. 225, p. 37. 
SOUSA, AGOSTINHO, OLIVEIRA, ARGENTIM, C OLIVEIRA, WECHSLERE AGOSTINHO

frequency of feeding on voluntary feed intake of Colossoma macropomum (Cuvier). Aquac. Res., 28: 419-432.

Mello, G.L. e Farias, A.P. 2007. Policultivo de tilápias e camarões marinhos: Os resultados das primeiras experiências em Laguna-SC. $\mathrm{Pa}$ norama da Aqüicultura, 17: 42-47.

Mello, S.C.R.P. 2001. Sistema inundado de criação de rãs. Ensaios experimentais. I Ciclo de Palestras Sobre Ranicultura do Instituto de Pesca. http://www.pesca.sp.gov.br/boletim_tec_ 31.htm. Data da consulta: outubro de 2007.

Ranário Aurora. 1938. Cultura da rã-gigante touro, Rana catesbeiana. Editora Ranário Aurora. Rio de Janeiro, 58 pp.

Toguyeni, A., Fauconneau, B., Boujard, T., Fostier,
A., Kuhn, E.R., Mol., K.A. and Baroiller, J.-F. 1997. Feeding behavior and food utilization in tilapia, Oreochromis niloticus: Effect of sex ratio and relationship with the endocrine status. Physiol. Behav., 62: 273-279.

Urbinati, E.C. e Carneiro, P.C.F. 2004. Práticas de manejo e estresse dos peixes em piscicultura. Em: Tópicos especiais em piscicultura de água doce tropical intensiva. Aquabio, 1: 171-194.

Vieira, J.S., Logato, P.V.R.L., Freitas, R.T.F. e Fialho, E.T. 2000. Efeito da densidade de estocagem no desempenho de tilapias do nilo (Oreochromis niloticus) em tanques-rede. Em: Reunião Anual da Sociedade Brasileira de Zootecnia 37. Viçosa, MG. Anais... Sociedade Brasileira de Zootecnia. Viçosa, MG.

Archivos de zootecnia vol. 59, núm. 225, p. 38. 